\title{
On A Subclass of Meromorphic Starlike Univalent Functions With Alternating Coefficients
}

\author{
T. Srinivas, P. Thirupathi Reddy, B. Madhavi \\ Department of Mathematics, Kakatiya University, Warangal- 506009 (A.P.) INDIA.
}

Abstract: Coefficient inequalities and distortion theorems are obtained for certain subclass of meromorphic starlike univalent functions with alternating coefficients. Further class preserving integral operators are obtained.

2000 Mathematics subject classification: 30 C 45.

Keywords: Regular, meromorphic, starlike, distortion theorem.

Let $\sum$ denote the class of functions of the form

\section{Introduction}

$$
f(z)=\frac{1}{z}+\sum_{m=1}^{\infty} a_{m} z^{m}
$$

which are regular in $E=\{z: 0<|z|<1\}$.

Define

$$
\begin{aligned}
& D^{0} f(z)=f(z) \\
& D^{1} f(z)=\frac{1}{z}+3 a_{1} z+4 a_{2} z^{2}+\ldots=\frac{\left(z^{2} f(z)\right)^{\prime}}{z} \\
& D^{2} f(z)=D\left(D^{\prime} f(z)\right)
\end{aligned}
$$

and for $\mathrm{n}=1,2,3, \ldots \ldots$

$$
D^{n} f(z)=D\left(D^{n-1} f(z)\right)=\frac{1}{z}+\sum_{m=1}^{\infty}(m+2)^{n} a_{m} z^{m}=\frac{\left(z^{2} D^{n-1} f(z)\right)^{\prime}}{z} .
$$

In [ 9 ] Uralegaddi and Somanatha obtained a new criteria for meromorphic starlike univalent functions via the basic inclusion relationship $B_{n+1}(\alpha) \subset B_{n}(\alpha),(0 \leq \alpha<1), n \in N_{0}=\{0,1,2, \ldots\}$, where $B_{n}(\alpha)$ is the class consisting of functions in $\sum$ satisfying

$$
\operatorname{Re}\left\{\frac{D^{n+1} f(z)}{D^{n} f(z)}-2\right\}<-\alpha,|z|<1,(0 \leq \alpha<1), n \in N_{0}=\{0,1,2, \ldots\} .
$$

We note that $B_{0}(\alpha)=\sum^{*}(\alpha)$, is the class of meromorphically starlike functions of order $\alpha(0 \leq \alpha<1)$, and $B_{0}(0)=\sum^{*}$ is the class of meromorphically starlike functions. Let $\sigma_{A}$ be the subclass of $\sum$ which consists of functions of the form

$$
f(z)=\frac{1}{z}+a_{1} z-a_{2} z^{2}+a_{3} z^{3}-\ldots . .=\frac{1}{z}+\sum_{m=1}^{\infty}(-1)^{m-1} a_{m} z^{m}, a_{m} \geq 0
$$

Further let $\sigma_{A, n}^{*}(\alpha, \beta)=B_{n}(\alpha, \beta) \cap \sigma_{A}$.

Definition1: Let $f(z)$ be defined by (1.3). Then $f(z) \in \sigma_{A, n}^{*}(\alpha, \beta)$ if and only

$\left|\frac{\frac{D^{n+1} f(z)}{D^{n} f(z)}-1}{\frac{D^{n+1} f(z)}{D^{n} f(z)}+2 \alpha-3}\right|<\beta$ for $|z|<1,0 \leq \alpha<1,0<\beta \leq 1$. 
On A Subclass Of Meromorphic Starlike Univalent Functions With Alternating Coefficients

In the present paper coefficient inequalities, distortion theorem and closure theorems for the class

$\sigma_{A, n}^{*}(\alpha, \beta)$ are obtained.Techniques used are similar to those Silverman [ 8 ].Finally, the class preserving integral operators of the form

$$
F(z)=\frac{c}{z^{c+1}} \int_{0}^{z} t^{c} f(t) d t \quad(c>0) .
$$

\section{Coefficient Inequalities}

Theorem 1.Let $f(z)=\frac{1}{z}+\sum_{m=1}^{\infty} a_{m} z^{m}$.If

$\sum_{m=1}^{\infty}(m+2)^{n}[(1+\beta) m+(2 \alpha-1) \beta+1]\left|a_{m}\right| \leq 2 \beta(1-\alpha)$

Then $f(z) \in B_{n}(\alpha, \beta)$.

Proof: Suppose that equation (2.1) holds for all admissable values of $\alpha, \beta$ and $n$. Consider the expression

$$
H\left(f, f^{\prime}\right)=\left|D^{n+1} f(z)-D^{n} f(z)\right|-\beta\left|D^{n+1} f(z)+(2 \alpha-3) D^{n} f(z)\right|
$$

Replacing $D^{n} f(z)$ and $D^{n+1} f(z)$ by their series expansions, we have, for $0<|z|=r<1$,

$$
\begin{aligned}
H\left(f, f^{\prime}\right) & =\left|\sum_{m=1}^{\infty}(m+2)^{n}(m+1) a_{m} z^{m+1}\right|-\beta\left|2(1-\alpha)-\sum_{m=1}^{\infty}(m+2)^{n}(m-1+2 \alpha) a_{m} z^{m+1}\right| \\
& \leq \sum_{m=1}^{\infty}(m+2)^{n}(m+1)\left|a_{m}\right| r^{m+1}-\beta\left\{2(1-\alpha)-\sum_{m=1}^{\infty}(m+2)^{n}(m-1+2 \alpha)\left|a_{m}\right| r^{m+1}\right\}
\end{aligned}
$$

Since the above inequality holds for all $\mathrm{r}(0<\mathrm{r}<1)$, letting $r \rightarrow 1$, we have

$$
\begin{aligned}
H\left(f, f^{\prime}\right) & \leq \sum_{m=1}^{\infty}(m+2)^{n}[(1+\beta) m+(2 \alpha-1) \beta+1]\left|a_{m}\right|-2 \beta(1-\alpha) \\
& \leq 0, \text { by (2.1). }
\end{aligned}
$$

Hence it follows that

$$
\left|\frac{D^{n+1} f(z)}{D^{n} f(z)}-1\right|<\left|\frac{D^{n+1} f(z)}{D^{n} f(z)}+(2 \alpha-3)\right|,
$$

which shows that $f(z) \in B_{n}(\alpha, \beta)$. Hence the Theorem is completely proved. For functions in $\sigma_{A, n}^{*}(\alpha, \beta)$ the converse of the above Theorem is also true.

Theorem 2. A function $f(z)$ in $\sigma_{A}$ is in $\sigma_{A, n}^{*}(\alpha, \beta)$ if and only if

$$
\sum_{m=1}^{\infty}(m+2)^{n}[(1+\beta) m+(2 \alpha-1) \beta+1] a_{m} \leq 2 \beta(1-\alpha) .
$$

Proof: In view of Theorem 1, it is sufficient to prove that only if part. Let us assume that $f(z)$ is in $\sigma_{A, n}^{*}(\alpha, \beta)$. Then

$$
\left|\frac{\frac{D^{n+1} f(z)}{D^{n} f(z)}-1}{\frac{D^{n+1} f(z)}{D^{n} f(z)}+2 \alpha-3}\right|=\left|\frac{\sum_{m=1}^{\infty}(-1)^{m-1}(m+2)^{n}(m+1) a_{m} z^{m+1}}{2(1-\alpha)-\sum_{m=1}^{\infty}(-1)^{m-1}(m+2)^{n}(m-1+2 \alpha) a_{m} z^{m+1}}\right|<\beta .
$$

Using the fact that $\operatorname{Re}(z) \leq|z|$ for all $z$,it follows that 
$\operatorname{Re}\left\{\frac{\sum_{m=1}^{\infty}(-1)^{m-1}(m+2)^{n}(m+1) a_{m} z^{m+1}}{2(1-\alpha)-\sum_{m=1}^{\infty}(-1)^{m-1}(m+2)^{n}(m-1+2 \alpha) a_{m} z^{m+1}}\right\}<\beta, \quad z \in E$.

Now choose the values of $\mathrm{z}$ on the real axis so that $\left(\frac{D^{n+1} f(z)}{D^{n} f(z)}-2\right)$ is real.Upon clearing the denominator in (2.4) and letting $z \rightarrow-1$ through real values, we obtain

$$
\begin{aligned}
& \sum_{m=1}^{\infty}(m+2)^{n}(m+1) a_{m} \leq \beta\left[2(1-\alpha)-\sum_{m=1}^{\infty}(m+2)^{n}(m-1+2 \alpha) a_{m}\right] \\
& \sum_{m=1}^{\infty}(m+2)^{n}\{(1+\beta) m+(2 \alpha-1) \beta+1\} a_{m} \leq 2 \beta(1-\alpha) .
\end{aligned}
$$

This completes the proof of the Theorem.

Corollary 1.Let the function $f(z)$ defined by $(1.3)$ be in the class $\sigma_{A, n}^{*}(\alpha, \beta)$.Then

$$
a_{m} \leq \frac{2 \beta(1-\alpha)}{(m+2)^{n}[(1+\beta) m+(2 \alpha-1)+1]}, \mathrm{m}=1,2,3, \ldots \ldots
$$

Equality holds for the function of the form

$$
f_{m}(z)=\frac{1}{z}+(-1)^{m-1} \frac{2 \beta(1-\alpha)}{(m+2)^{n}[(1+\beta) m+(2 \alpha-1)+1]} z^{m}
$$

\section{Distortion Properties and Radius of Convexity}

Theorem 3. Let the function $f(z)$ defined by $(1.3)$ be in the class $\sigma_{A, n}^{*}(\alpha, \beta)$. Then for $0<|z|=r<1$,

$$
\frac{1}{r}-\frac{\beta(1-\alpha)}{3^{n}(1+\alpha \beta)} r \leq|f(z)| \leq \frac{1}{r}+\frac{\beta(1-\alpha)}{3^{n}(1+\alpha \beta)} r
$$

with equality for the function

$$
f(z)=\frac{1}{z}+\frac{\beta(1-\alpha)}{3^{n}(1+\alpha \beta)} z, \quad \text { at } \mathrm{z}=\mathrm{r}, \text { ir . }
$$

Proof: Suppose $f(z)$ is in $\sigma_{A, n}^{*}(\alpha, \beta)$. In view of Theorem 2, we have

$$
\sum_{m=1}^{\infty} a_{m} \leq \frac{\beta(1-\alpha)}{3^{n}(1+\alpha \beta)}
$$

Then for $0<|z|=r<1$,

$$
\begin{aligned}
|f(z)|=\mid \frac{1}{z}+\sum_{m=1}^{\infty}( & -1)^{m-1} a_{m} z^{m} \mid \\
& \leq\left|\frac{1}{z}\right|+\sum_{m=1}^{\infty} a_{m}|z|^{m} \leq \frac{1}{r}+r \sum_{m=1}^{\infty} a_{m} \\
& \leq \frac{1}{r}+\frac{\beta(1-\alpha)}{3^{n}(1+\alpha \beta)} r
\end{aligned}
$$


This gives the right hand side inequality of (3.1).

Also,

$$
\begin{aligned}
|f(z)| & =\left|\frac{1}{z}+\sum_{m=1}^{\infty}(-1)^{m-1} a_{m} z^{m}\right| \\
& \geq\left|\frac{1}{z}\right|-\sum_{m=1}^{\infty} a_{m}|z|^{m} \\
& \geq \frac{1}{r}-r \sum_{m=1}^{\infty} a_{m} \\
& \geq \frac{1}{r}-\frac{\beta(1-\alpha)}{3^{n}(1+\alpha \beta)} r
\end{aligned}
$$

which gives the left hand side inequality of (3.1). This completes the proof. Putting $n=0$ and $\beta=1$ in the above Theorem, we have the following.

Corollary 2.Let the function $f(z)$ defined by $(1.3)$ be in the class $\sigma_{A, 0}^{*}(\alpha, 1)=\sigma_{A}^{*}(\alpha)$. Then for

$0<|z|=r<1$,

$$
\frac{1}{r}-\frac{(1-\alpha)}{(1+\alpha)} r \leq|f(z)| \leq \frac{1}{r}+\frac{(1-\alpha)}{(1+\alpha)} r
$$

The result is sharp.

We observe that our result in corollary 2 improves the result of Uralegaddi and Ganigi[10]

Theorem 4. Let the function $f(z)$ defined by ( 1.3$)$ be in the class $\sigma_{A, n}^{*}(\alpha, \beta)$. Then for

$0<|z|=r<1$,

$$
\frac{1}{r^{2}}-\frac{\beta(1-\alpha)}{3^{n}(1+\alpha \beta)} r \leq\left|f^{\prime}(z)\right| \leq \frac{1}{r^{2}}+\frac{\beta(1-\alpha)}{3^{n}(1+\alpha \beta)} r .
$$

The result is sharp,the extremal function being of the form (3.2).

Proof: From Theorem 2, we have

$3^{n}(1+\alpha \beta) \sum_{m=1}^{\infty} m a_{m} \leq \sum_{m=1}^{\infty}(m+2)^{n}\{(1+\beta) m+(2 \alpha-1) \beta+1\} a_{m} \leq \beta(1-\alpha)$

which evidently yields

$$
\sum_{m=1}^{\infty} m a_{m} \leq \frac{\beta(1-\alpha)}{3^{n}(1+\alpha \beta)}
$$

Consequently, we obtain

$$
\begin{array}{r}
\left|f^{\prime}(z)\right| \leq \frac{1}{r^{2}}+\sum_{m=1}^{\infty} m a_{m} r^{m-1} \leq \frac{1}{r^{2}}+\sum_{m=1}^{\infty} m a_{m} \\
\leq \frac{1}{r^{2}}+\frac{\beta(1-\alpha)}{3^{n}(1+\alpha \beta)} .
\end{array}
$$

Also,

$$
\left|f^{\prime}(z)\right| \geq \frac{1}{r^{2}}-\sum_{m=1}^{\infty} m a_{m} r^{m-1} \geq \frac{1}{r^{2}}-\sum_{m=1}^{\infty} m a_{m}
$$


This completes the proof.

\section{References}

[1] F. M. Al - Oboudi, On univalent functions defined by a generalized salagean operator, Internat J. Math. Math. Sci., 27(2004), 1429-1436.

[2] M. K. Aouf and H. M. Hossen, New criteria for meromorphic p - valent starlike functions, Tsukuba J. Math. 17(2)(1993), 481486.

[3] M. K. Aouf and H. E. Darwish, Meromorphic starlike univalent functions with alternating coefficients, Utilities Math. 47(1995), $137-144$.

[4] M. Darus, S. B. Joshi and N. D. Sangle, Meromorphic starlike functions with alternating and missing coefficients, General Mathematics (2006), $113-126$.

[5] H. E. Darwish, Meromorphic p-valent starlike functions with negative coefficients, Indian. J. Pure Appl. Math. 33(7), 2002, 967976.

[6] M. L. Mogra, T. R. Reddy and O. P. Juneja, Meromorphic univalent functions with positive coefficients, Bull. Austral. Math. Soc, 32(1985) 161-176.

[7] T. Ram Reddy and P. Thirupathi Reddy, Meromorphic p-valent

[8] starlike functions with alternating coefficients, Bull. Pure Appl.Math. Vol 3,(2009),254-262.

[9] H. Silverman, Univalent functions with negative coefficients, Proc. Amer. Math. Soc. 51(1975), 109-116

[10] B. A. Uralegeddi and C. Somanatha, New criteria for meromorphic starlike univalent functions, Bull. Austral. Math. Soc. 43(1991), 137-140.

[11] B. A. Uralegaddi and M. D. Ganigi, Meromorphic starlike functions

[12] with alternating coefficients, Rend. Math. 11(7) (1991), 441- 446. 